\title{
MASSIVE MIMO, MM WAVE AND 5G TECHNOLOGY INSIGHTS AND CHALLENGES
}

Sara Bhatti

Sir Syed University of Engineering \& Technology. Karachi (Pakistan) E-mail:sarab@ssuet.edu.pk

Recepción: 05/03/2019 Aceptación: 05/04/2019 Publicación: 17/05/2019

\section{Citación sugerida:}

Bhatti, S. (2019). Massive MIMO, MM wave and 5G Technology insights and challenges. 3C Tecnología. Glosas de innovación aplicadas a la pyme. Edición Especial, Mayo 2019, pp. 498517. doi: http://dx.doi.org/10.17993/3ctecno.2019.specialissue2.498-517

\section{Suggested citation:}

Bhatti, S. (2019). Massive MIMO, MM wave and 5G Technology insights and challenges. 3C Tecnología. Glosas de innovación aplicadas a la pyme. Special Issue, May 2019, pp. 498-517. doi: http://dx.doi.org/10.17993/3ctecno.2019.specialissue2.498-517 


\section{ABSTRACT}

The global broadband cellular demand is increasing exponentially resulting in a worldwide shortage of available bandwidths allocated for wireless transmission, leading to in-depth research being carried out in the underutilized Millimeter Wave (mmWave) bands which occupy the frequencies above $3 \mathrm{GHz}$ in the frequency spectrum. The $5 \mathrm{G}$ technology is evolving rapidly from the current $3 \mathrm{G}$ and $4 \mathrm{G}$ networks deployed worldwide, and the mmWave technology plays a vital role in the future $5 \mathrm{G}$ networks. Massive MIMO will be unprecedented during the design considerations when utilizing mmWave data streams with not only less complexity but will also enhance the spectral efficiency of the wireless system economically. This paper looks at the $5 \mathrm{G}$ revolution, its advantage over $4 \mathrm{G}$, the incorporation between $5 \mathrm{G}$ and mmWave technologies, and MIMO antenna design considerations. It will also highlight the challenges facing the above technologies, and some new technologies such as Ultra Dense Networks (UDN), smart cities and $\mathrm{Li}-\mathrm{Fi}$ which will incorporate mmWave, MIMO and $5 \mathrm{G}$ technology.

\section{KEYWORDS}

3GHz, Millimeter Wave, 3G, 4G, 5G, MIMO, UDN, Smart cities, Li-Fi. 


\section{INTRODUCTION}

The expeditious increase in the worldwide demand for high speed, secure, bi-directional and fully networked wireless communications has led forward vast research towards alternate frequencies in the spectrums. The limited bandwidth resources which are currently available are being exhausted due to the high Compound Annual Growth Rate (GAGR) of wireless traffic, and this has prompted research into the previously untapped high-frequency bands of $3 \mathrm{GHz}$ to $300 \mathrm{GHz}$ in the frequency spectrum as shown in Sharma (2013), also known as Millimeter Wave (mmWave) spectrum. This spectrum shares similar propagation characteristics and can be incorporated relatively easily with the wireless technologies currently deployed worldwide (Pi \& Khan, 2012).

\subsection{MMWAVE FREQUENCY ALLOCATION}

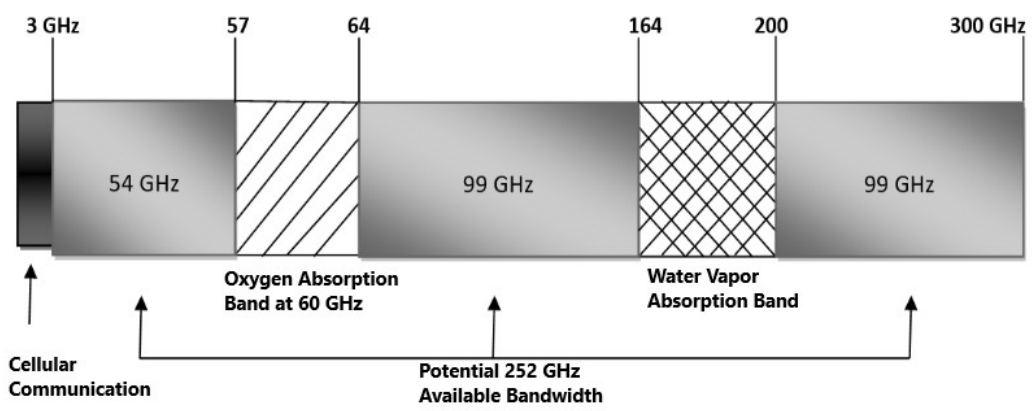

Figure 1. The frequency range of the mmWave (Pi \& Khan, 2012).

The mmWave range within the frequency spectrum is between $3 \mathrm{GHz}$ to $300 \mathrm{GHz}$, as illustrated in Figure 1. It is shown that the $57-64 \mathrm{GHz}$ band is limited to oxygen absorption and the $164-200 \mathrm{GHz}$ band is not suitable for propagation due to severe water vapor attenuation, with maximum losses at $180 \mathrm{GHz}$. Therefore, only around $252 \mathrm{GHz}$ of the spectrum is available for mobile broadband communication (Federal Communications Commission, 1997). If it is assumed that only $40 \%$ of the mmWave spectrum is available for mobile broadband communication, it still provides more than $100 \mathrm{GHz}$ of unused frequencies, which are about 200 times more than the current $4 \mathrm{GHz}$ frequency bands deployed worldwide (Pi \& Khan, 2012). 


\section{2. (5G) DEPLOYMENT WORLDWIDE}

Table 1. Frequency Allocations for Cellular Usage within different generations.

\begin{tabular}{|c|c|}
\hline \multicolumn{2}{|c|}{$1 G$} \\
\hline & $800 \mathrm{MHz}$ \\
\hline \multicolumn{2}{|c|}{$2 \mathrm{G}$} \\
\hline D AMPS & $800 \mathrm{MHz}-1.9 \mathrm{GHz}$ \\
\hline GSM & $800 \mathrm{MHz}-1.9 \mathrm{GHz}$ \\
\hline AS95 A/B & $800 \mathrm{MHz}-1.9 \mathrm{GHz}$ \\
\hline \multicolumn{2}{|c|}{$3 G$} \\
\hline UMTS & $2 \mathrm{GHz}$ \\
\hline WCDMA & $2 \mathrm{GHz}$ \\
\hline CDMA2000 & $2 \mathrm{GHz}$ \\
\hline \multicolumn{2}{|c|}{ 4G } \\
\hline LTE & $1.8-2.5 \mathrm{GHz}$ \\
\hline \multicolumn{2}{|c|}{$5 \mathbf{G}$} \\
\hline LTE & $600 \mathrm{MHz}-6 \mathrm{GHz}$ \\
\hline mmWave & $24-86 \mathrm{GHz}$ \\
\hline
\end{tabular}

As seen in Table 1, the current mobile networks which utilize their allotted radio frequencies to provide cellular services don't exceed $780 \mathrm{MHz}$, and each provider has about 200MHz of spectrum available to them (Rappaport, et al., 2013). This bandwidth is proving to be insufficient for the explosive traffic volumes, even for the most advanced $4 \mathrm{G}$ technologies.

To meet these unprecedented magnitudes in demands, 5G networks, which are still under research, are proving to be a promising solution and are expected to hit initial phases of commercialization by 2020, with global adoption by 2025 (Sharma, 2013). To reach the 5G design target, there are three paramount approaches: Ultra-dense Networks (UDN), large spectrum efficiency and increase bandwidth allocation. This is harnessed by mmWave incorporated with a large number of antennae, also referred to as mmWave massive Multiple Input Multiple Output (MIMO) antennae, to provide a wireless network with high speeds and smaller cell sizes to support not only the cellular demands, but also backhaul and Wi-Fi services (Rappaport, et al., 2013). 


\subsection{G EVOLUTION}

Each new cellular generation, when compared to the previous generation, is an enhancement the system electronics, security, speed, frequency, data capacity and latency. This journey began with $1 \mathrm{G}$, followed by $2 \mathrm{G}, 3 \mathrm{G}$, the currently deployed $4 \mathrm{G}$ and the newly emerging $5 \mathrm{G}$ technologies. A new generation has appeared every ten years since 1981 and has followed its own evolutionary path towards achieving higher speeds and better performance, as the global market of mobile and wireless communication has increased exponentially.

The first generation $(1 \mathrm{G})$ used the analog transmission to fulfill basic mobile voice transmission. The $2 \mathrm{G}$ systems used digitally enhanced multiple access technologies such as TDMA (Time Division Multiple Access) and CDMA (Code Division Multiple Access) leading towards early data services and enhanced spectral efficiency, under the Enhanced Data for Global Evolution (EDGE) standard. However, the standards were found to differ globally, and a network was developed where the design standards would not differ and be independent of the technology platform. And hence 3G was implemented (Sharma, 2013; Swindlehurst, Ayanoglu, Heydari \& Capolino, 2014).

In 3G, technologies such as Wideband Code Division Multiple Access (W-CDMA) and High-Speed Packet Access (HSPA) resulted in enhanced improvements within video and audio streaming capabilities (Rappaport, et al., 2013) by supporting information transfer rate of at least 2Mbps.

$3 \mathrm{G}$ was a family of standards working together to meet the IMT-2000 technical standards (Bangerter, Talwar, Arefi, \& Stewart, 2014). Universal Terrestrial Mobile System (UMTS) was adopted in Europe, while the American 3G technology is named as cdma2000 (Hossain, 2013) and both were developed by the Third Generation Partnership Project (3GPP).

3GPP also developed the Long-Term Evolution(LTE) to offer a complete 4G capable mobile broadband and an upgrade to the existing $3 \mathrm{G}$ network. LTE uses Orthogonal Frequency-Division Multiplexing (OFDM) to support a transmission bandwidth 
of $20 \mathrm{MHz}$, while also supporting MIMO antenna arrays. These combined with dynamic channel allocation and channel-dependent scheduling allows for the utilization of propagation via multiple paths, in order to improve signal performance, spectral efficiency and diversity (Bangerter, Talwar, Arefi \& Stewart, 2014).

The predicted increase in mobile broadband demands is up to a thousand fold by the year 2020, which has led to the motivation behind research in alternate spectrums beyond the $4 \mathrm{G}$ standard.

$5 \mathrm{G}$ is the latest generation to be developing in the wireless revolution. It promises speeds of up to $10 \mathrm{Gbps}, 100$ times faster than $4 \mathrm{G}$. It has low latency of $1 \mathrm{~ms}$ or less, and mobility with larger coverage areas. The higher data rates will allow services beyond cell phones, and base stations will provide the necessary bandwidth for office and home usage, which was not possible in previous generations (Rappaport, et al., 2013).

\subsection{G AND MMWAVE INCORPORATION}

Currently, the $700 \mathrm{MHz}$ to $2.6 \mathrm{GHz}$ radio spectrum is highly saturated for all wireless applications, and the $5 \mathrm{G}$ mmWave technological advances will support the imminent congestion by providing at least $100 \mathrm{GHz}$ of bandwidth which is substantially greater and would allow 4G service providers to expand their channel bandwidths significantly.

The incorporation of $5 \mathrm{G}$ with the mmWave spectrum will also allow antennae with highly directional beam-forming at the base stations and mobile phones, lower infrastructure costs due to smaller base stations, longer battery life and provisions of uniform, uninterrupted and consistent connectivity.

This will be achieved with the usage of steerable antenna arrays as the mmWave spectrum will simultaneously support mobile communications as well as backhaul, and possibly allow for the convergence of both cellular and $\mathrm{Wi}-\mathrm{Fi}$ services (Swindlehurst, et al., 2014). The smaller wavelengths allow for physically smaller antenna arrays, which is discussed in detail in the following section. 
Recent developments into CMOS technology operating within the mmWave spectrum, as well as antennas that are steerable both at the base station as well as the mobile phone has further enhanced the operation of $5 \mathrm{G}$ within this spectrum (Gutierrez, Agarwall, Parrish \& Rappaport, 2009). The smaller component sizes, about 40nm, allows them to be applicable in numerous areas such as radar transceiver and in chipsets employed in automotive and industrial applications.

The higher frequencies and bandwidth allocations in the mmWave spectrum allow for a significant increase in data transfers. This, in turn, reduces latency, which is invaluable for digital internet-based applications and access requiring minimal latency.

The mmWave spectral band results in smaller cell sizes as RF path loss increases with frequency. This increase can be overcome by the large beamforming gains obtained through massive antenna arrays, and simultaneously increase the coverage areas (Swindlehurst, et al., 2014). Operating in smaller cells reduce the channel coherence time and allow higher channel coherence bandwidth and lower mobility. All these factors, as well as the smaller wavelengths and higher frequencies, resulting in a reduction in the antenna sizes and electronics, which are appealing for massive MIMO transceiver design (Swindlehurst, et al., 2014).

As compared to the $4 \mathrm{G}$ networks in populous areas, mmWaves offer such a high jump in bandwidth that links between station-to-device, as well as backhaul, can be established wirelessly and would be capable to handle the larger traffic. Using smart, steerable antennas would allow the base station cost to be reduced as their numbers would increase in urban environments, thus increasing wireless backhaul even more achievable and necessary (Rappaport, et al., 2013).

\section{MASSIVE MIMO AND 5G}

Millimeter-wave (mmWave) (30-300 GHz) Multiple-Input Multiple-Output (MIMO) with large antenna array, incorporating 5G technology, has been considered as a promising solution to meet the one thousand times increase in data traffic predicted in the near future (Marzetta, 2010). Besides providing 
larger bandwidth compared to the current $4 \mathrm{G}$ wireless communications, antenna arrays can now be packed into a smaller physical size due to the shorter wavelengths associated with mmWave frequencies. This means that the spectral efficiency can be improved considerably as MIMO with a large antenna array effectively compensate for the high path loss induced by high frequencies (Hossain, 2013).

To increase the diversity or the number of degrees of freedom in the wireless communication system, the usage of multiple antennas is essential. Compared to the conventional single antenna channels, a system with multiple transmits and receive antennas (MIMO) has higher spectral efficiency and higher bandwidth, as each pair of transmitting and receiving antennas will provide separate signal paths from the transmitter to receiver.

Reception reliability is therefore increased as signals carrying the same information are transmitted on different paths, and multiple independently faded replicas of the data symbols are received at the receiver (Zheng \& Tse, 2003). Therefore, the probability that all signal symbols fade simultaneously reduces as the receiver receives multiple independently faded replicas of the same information symbol.

At higher frequencies, rain, foliage and atmospheric absorption are a serious impediment to mmWave mobile communication. The attenuation caused by rain and oxygen absorption is around $10-20 \mathrm{~dB} / \mathrm{km}$. These issues can be overcome when we consider that the cell sizes have been reduced to about 50$200 \mathrm{~m}$, especially at $28 \mathrm{GHz}$ and $38 \mathrm{GHz}$, equating to about $1.4 \mathrm{~dB}$ over $200 \mathrm{~m}$ (Rappaport, et al., 2013; Gao, Dai, Mi, Wang, Imran \& Shakir, 2015).

The primary imposing factor on the cell size in mmWave massive MIMO would be free space path loss. In smaller cells, this loss can limit intercell interference, and allow for greater frequency reuse.

In a wireless communication downlink system, the relationship between Transmitted Power $\left(P_{t}\right)$, Received Power $\left(P_{r}\right)$, Transmitted Gain $\left(G_{t}\right)$ of entire transmit array and Received Gain $\left(\mathrm{G}_{\mathrm{r}}\right)$ is given by 


$$
P_{r}=\frac{P_{t} G_{t} G_{r}}{(4 \pi r)^{2} \lambda^{2}}
$$

In reference to Eq. (1), the path loss is given by $(4 \pi r)^{2} \lambda^{2}$, and is directly proportional to wavelength, $\lambda$, where $r$ is the range (Zheng \& Tse, 2003). A larger path loss value can be compensated by increasing the transmitted power, receiver sensitivity, antenna array gain and reducing channel noise. Hence, equipping massive antennas at macro and small-cell base--stations (BSs) can not only compensate for severe path loss whilst achieving larger coverage distances, but also improve signal directivity. Deploying antenna arrays with a large number of antennas will increase the antenna gain (Gao, et al., 2018).

To achieve dramatic gains, MIMO envisions BSs with antennas numbering 100 or more, and this concept leads to massive MIMO. BSs with a very large number of antennas $\mathrm{N}_{\mathrm{t}}$, serve a group of single antenna $\mathrm{co}^{-}$-channel users. It can be concluded that as $\mathrm{N}_{\mathrm{t}}$ approaches infinity, where throughput and quantity of terminals are independent of cell size, the effects of uncorrelated noise and fast fading vanish and the spectral efficiency is independent of bandwidth, with the required transmitted energy per bit vanishes (Swindlehurst, et al., 2014; Marzetta, 2010).

A larger $\mathrm{N}_{\mathrm{t}}$ enables a substantial increase in capacity, but in reality causes interference problems which can be alleviated by using beamforming antennas as opposed to the conventional ones (Ali, Ismail, Nordin \& Abdulah, 2017).

In MIMO, beamforming is a signal processing procedure where the radiated beam patterns of the antenna are produced by formulating the processed signal in the direction of the desired terminals while cancelling interfering signals. This enhances the energy efficiency, spectral efficiency while increasing system security (Jungnickel, et al., 2014).

As the value of $\mathrm{N}_{\mathrm{t}}$ increases, more orthogonal pilots are required for channel estimation, which is exhausting for the radio resources $(\mathrm{Lu}, \mathrm{Li}$, Swindlehurst, Ashikhmin \& Zhang, 2014). As the number of independent pilot sequences is limited, they are reused between different cells and can cause conflicts in antenna 
arrays known as pilot contamination (Jungnickel, et al., 2014). Pilot contamination in massive MIMO systems limits the spectral efficiency and increases with an increase in $\mathrm{N}_{\mathrm{t}}$.

It is assumed that as $\mathrm{N}_{\mathrm{t}}$ increases, the user channels remain spatially uncorrelated and remain orthogonal with their respective pairs given proper propagation conditions. However, increasing the number of antennas cannot provide orthogonality in the highly correlated channels and user scheduling can render a problem and signal processing of higher complexity is needed to separate spatially correlated users (Ali, et al., 2017).

\section{APPLICATIONS OF 5G}

As wireless access techniques are being deployed extensively due to the rapid convergence of computational technologies and information communication, $5 \mathrm{G}$ can no longer be constricted to conventional technical characteristics. 5G is a cloud of multiple technologies, a diverse system of air interfaces, frequency bands, protocols, network types and access node classes which are integrated to provide smart and customized services, ranging from medicine to smart cities and the more obvious cellular technologies. $5 \mathrm{G}$ should not be viewed as a solution to replacing the current $4 \mathrm{G}$ LTE systems, but the seamless and efficient utilization of advanced technologies and spectrum-usage schemes.

\subsection{ULTRA-DENSE RADIO ACCESS NETWORKS}

Ultra-Dense Radio Access Networks (UDRANETS) is seen as a promising system architecture to enable high traffic capacity over extremely reliable shortrange links (Chávez-Santiago, et al., 2015). 


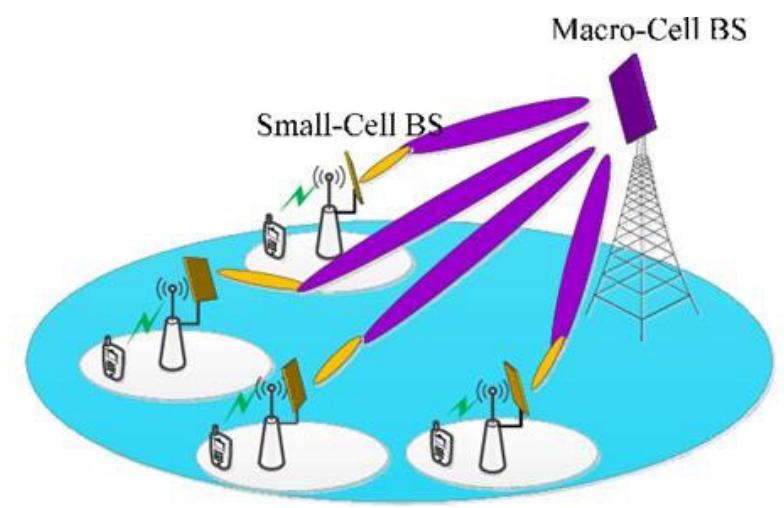

Figure 2. MmWave massive MIMMO based 5G UDN (Skouby \& Lynggaard, 2014).

A macro-cell base station with large coverage areas is responsible for the user scheduling and resource allocation of high mobility users, while the various ultra-dense smaller BSs provide a very high data rate for low mobility users (Gao, et al., 2015), as illustrated in Figure 2. The UDRANETS allow for better frequency reuse and improving energy efficiency and reduction in path loss. There is a possibility of completely wireless backhaul based on the mmWave massive MIMO in the future (Sulyman, et al., 2014).

These cells do pose several problems, such as frequent handovers due to small cell sizes, higher energy consumption and backhaul, which will increase interference and mobility (Hao, Yan, Yu-Ngok \& Yuan, 2016).

\subsection{SMART CITIES AND $5 G$}

The deployment of new ecosystems utilizing $5 \mathrm{G}$ technologies for the combination of smart cities and homes has huge potential, with a focus on compatibility with existing infrastructures to create sustainable and cost-efficient urban environments. This will provide multiple options in a smart city in areas of health care, green ecosystems and intelligent community services such as e-businesses, security surveillance, social networks, intelligent transportation, telemedicine, logistical management, Internet of things (IoT) and Cloud of Things (CoT), Artificial Intelligence (AI) just to name a few (Lynggaard \& Skouby, 2015). 
Smart homes are targeted towards enriching the living environment and improving the quality of life of its inhabitants. This targets the home automation area, where embedded devices such as light, heating, entertainment systems security are controlled remotely (Skouby \& Lynggaard, 2014).

The wireless technologies such as Bluetooth, Radio Frequency Identification (RFID), ZigBee, Wireless Local Area Networks (WLANs), sensor networks along with fiber communication and cable networks form the basis of smart city infrastructures (Han, Ge, Wang, Kwak, Han \& Liu, 2017). All these together with IoT produces a huge amount of information that needs to be securely stored for processing. Hence, the incorporation of CoT with rest of these technologies is crucial as it provides resources and calculation capabilities which are accessible via the internet and can store the IoT data efficiently.

There are various challenges that face the development of such ambitious technologies. The complexity of the movement of various mobile terminals increases and becomes more varied. The data transmission can be of a pedestrian with a cellular phone, a laptop device on a high-speed train or even a navigational device on a moving car. There are also various obstacles in a dense urban environment, especially in the mmWave spectrum.

The security and privacy of networks users pose a serious threat to the development of $5 \mathrm{G}$ technologies. Sensitive and personal information such as banking information would have to be shared on a cloud platform and could pose a threat of being disclosed to the wrong persons. This would require highly complex data security management with strong encryption and cryptographic tools to maintain network system confidentiality and also to identify vulnerabilities in networks that could serve as weak points for various attacks (Gao, et al., 2015; Mehmood, et al., 2017). 


\subsection{LI-FI AND 5G}

High--speed wireless data communication using infrared and visible light spectrum is being deployed and is predominantly known as $\mathrm{Li}-\mathrm{Fi}$. The concept of Visible Light Communication (VLG) is used to achieve bi-directional high-speed, secure and fully networked wireless communication.

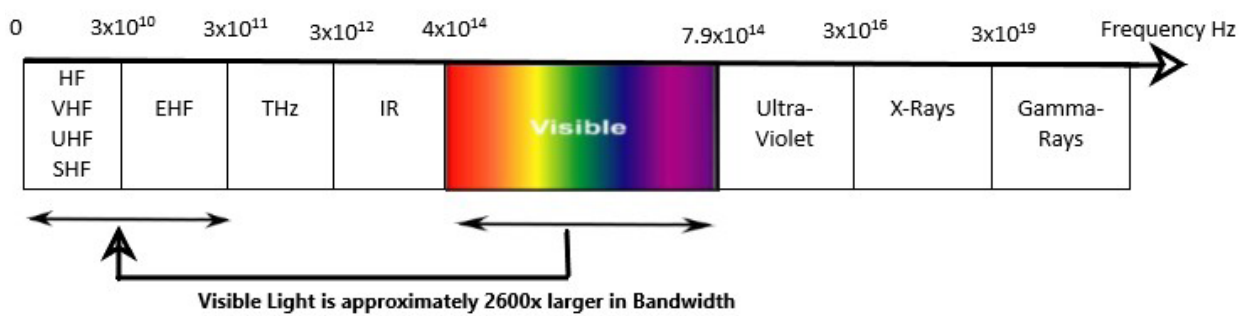

Figure 3. Comparison of the Radio Frequency (RF) Spectrum and the Visible Light Spectrum (Haas, 2018).

As illustrated in Figure 3, the bandwidth occupied by VLC is approximately 2600 times greater than the currently employed radio frequencies for wireless communication. The conventional VLC system was being conceived as a single point-to-point wireless communication between a LED source and a receiver that is equipped with a photodetection device. The data rates required to depend on digital modulation and lighting technology.

Adoption of wireless networks based on light as opposed to pointing to point links pose several challenges. As each cell can have several users, multiple access schemes are required. Uplink provisions can be the difference from downlink ones, as the portable device requires lower energy consumption, and the user is not likely to be distracted by the visible light source (Swindlehurst, et al., 2014), and therefore infrared spectrum is the most desirable for uplink (Bangerter, et al., 2014).

The high-speed uplink requires its modulation to be both power and spectrum efficient simultaneously. Handovers will be complicated as connectivity needs to be maintained as a user leaves a certain premise for an area without any $\mathrm{Li}-\mathrm{Fi}$ coverage (Ayyash, et al., 2016). 
However, the incorporation between $\mathrm{Wi}-\mathrm{Fi}$ and $\mathrm{Li}-\mathrm{Fi}$ proves promising and it is a possibility for the two of them to co-exist. It will allow for off-loading opportunities for the Wi-Fi network as immobile users would be utilizing the $\mathrm{Li}-$ Fi technology. With this collaboration, the total number of users can more than triple, and throughput can increase exponentially, by enhancing indoor coverage and providing high data rates.

\section{CONCLUSION}

To achieve dramatic demands that wireless technologies will need in terms of capacity and spectral efficiency, 5G systems embracing mmWave spectrum from $3300 \mathrm{GHz}$ is promising. It promises high speed, low latency amongst other advantages, and would meet the predicted demands that $4 \mathrm{G}$ networks will meet by 2020. It will result in smaller cell sizes, and wireless backhauls to be implemented. Higher spectrums allow for utilization of highly directional antennas to be packed into larger arrays with smaller physical sizes. This is appealing for the massive MIMO transceiver design. To reduce the path loss mmWaves experience, increasing the number of antennas can lead to high capacity, but also high interference. Signal analysis complexity increases, and results in pilot contamination.

The Ultra-Dense Radio Access Networks (UDRANETS) is a system architecture to meet high traffic and handle large capacity. $5 \mathrm{G}$ technologies are being deployed in smart cities, embracing IoT and CoT technologies. This would meet some challenges such as data security, privacy and an increase in overall signal analysis complexity.

$\mathrm{Li}-\mathrm{Fi}$ would be a promising solution as it would be able to integrate with $\mathrm{Wi}-\mathrm{Fi}$ and increase the capacity, while also enhancing indoor and outdoor coverage. 


\section{REFERENCES}

Ali, E., Ismail, M., Nordin, R. \& Abdulah, N. F. (2017). Beamforming techniques for massive MIMO systems in 5G: overview, classification, and trends for future research. Frontiers of Information Technology E Electronic Engineering, 18(6), pp. 753-772. doi: http://dx.doi.org/10.1631/fitee.1601817

Ayyash, M., Elgala, H., Khreishah, A., Jungnickel, V., Little, T., Shao, S., ... Freund, R. (2016). Coexistence of WiFi and LiFi toward 5G: concepts, opportunities, and challenges. IEEE Communications Magazine, 54(2), pp. 64-71. doi: http://dx.doi.org/10.1109/MCOM.2016.7402263

Bangerter, B., Talwar, S., Arefi, R. \& Stewart, K. (2014). Networks and devices for the $5 \mathrm{G}$ era. IEE Comunications magazine, 52(2), pp. 90-96. doi: http:// dx.doi.org/10.1109/MCOM.2014.6736748

Chávez-Santiago, R., Szydełko, M., Kliks, A., Foukalas, F., Haddad, Y., Nolan, K. E., ... Balasingham, I. (2015). 5G: The convergence of wireless communications. Wireless Personal Communications, 83(3), pp. 1617-1642. doi: http://dx.doi.org/10.1007/s1 1277-015-2467-2

Gao, X., Dai, L., \& Sayeed, A. M. (2018). Low RF-complexity technologies to enable millimeter-wave MIMO with large antenna array for $5 \mathrm{G}$ wireless communications. IEEE Communications Magazine, 56(4), pp. 211-217. doi: http:// dx.doi.org/10.1109/MCOM.2018.1600727

Gao, Z., Dai, L., Mi, D., Wang, Z., Imran, M. A., \& Shakir, M. Z. (2015). MmWave massive-MIMO-based wireless backhaul for the $5 \mathrm{G}$ ultra-dense network. IEEE Wireless Communications Magazine, 22(5), pp. 13-21. doi: http:// dx.doi.org/10.1109/MWC.2015.7306533

Gutierrez, F., Agarwall, S., Parrish, K. \& Rappaport, T. S. (2009). Onchip integrated antenna structures in CMOS for $60 \mathrm{GHz}$ WPAN systems. IEEE fournal on Selected areas in Communications, 27(8), pp. 1367-1378. 
Haas, H. (2018). LiFi is a paradigm-shifting 5G technology. Reviews in Physics, 3, pp. 26-31.

Han, T., Ge, X., Wang, L., Kwak, K. S., Han, Y. \& Liu, X. (2017). 5G converged cell-less communications in smart cities. IEEE Communications Magazine, 55(3), pp. 44-50. doi: http://dx.doi.org/10.1109/MCOM.2017.1600256CM

Hao, P., Yan, X., Yu-Ngok, R. \& Yuan, Y. (2016). Ultra dense network: Challenges enabling technologies and new trends. China Communications, 13(2), pp. 30-40. doi: http://dx.doi.org/10.1109/CG.2016.7405723

Hossain, S. (2013). 5G wireless communication systems. American fournal of Engineering Research (A7ER), 2(10), pp. 344-353.

Jungnickel, V., Manolakis, K., Zirwas, W., Panzner, B., Braun, V., Lossow, M., . . . Svensson, T. (2014). The role of small cells, coordinated multipoint, and massive MIMO in 5G. IEEE Communications Magazine, 52(5), pp. 44-51. doi: http://dx.doi.org/10.1109/MCOM.2014.6815892

Lu, L., Li, G. Y., Swindlehurst, A. L., Ashikhmin, A. \& Zhang, R. (2014). An overview of massive MIMO: Benefits and challenges. IEEE Journal of Selected Topics in Signal Processing, 8(5), pp. 742-758. doi: http://dx.doi.org/10.1109/ JSTSP. 2014.2317671

Lynggaard, P. \& Skouby, K. E. (2015). Deploying 5G-technologies in smart city and smart home wireless sensor networks with interferences. Wireless Personal Communications, 81(4), pp. 1399-1413. doi: http://dx.doi.org/10.1007/s11277015-2480-5

Marzetta, T. (2010). Noncooperative cellular wireless with unlimited numbers of base station antennas. IEEE Transactions on Wireless Communications, 9(11), p. 3590. doi: http://dx.doi.org/10.1109/TWC.2010.092810.091092 
Mehmood, Y., Ahmad, F., Yaqoob, I., Adnane, A., Imran, M., \& Guizani, S. (2017). Internet-of-things-based smart cities: Recent advances and challenges. IEEE Communications Magazine, 55(9), pp. 16-24. doi: http:/ / dx.doi.org/10.1109/ MCOM.2017.1600514

Pi, Z. \& Khan, F. (2012). A millimeter-wave massive MIMO system for next generation mobile broadband. In 2012 Conference Record of the Forty Sixth Asilomar Conference on Signals, Systems and Computers (ASILOMAR). doi: http://dx.doi.org/10.1109/ AC.SSC.2012.6489100

Federal Communications Commission. (1997). Spectrum Management Implications: Federal Communications Commission Office of Engineering and Technology. (70), p. 9.

Rappaport, T. S., Sun, S., Mayzus, R., Zhao, H., Azar, Y., Wang, K., ... Gutierrez, F. (2013). Millimeter wave mobile communications for 5G cellular: It will work!. IEEE Access, 1, pp. 335-349. doi: http://dx.doi.org/10.1109/ ACGESS.2013.2260813

Sharma, P. (2013). Evolution of mobile wireless communication networks-1G to $5 \mathrm{G}$ as well as future prospective of next generation communication network. International Fournal of Computer Science and Mobile Computing, 2(8), pp. 47-53.

Skouby, K. E. \& Lynggaard, P. (2014). Smart home and smart city solutions enabled by 5G, IoT, AAI and CoT services. In 2014 International Conference on Contemporary Computing and Informatics (IC3I). doi: http://dx.doi.org/10.1109/ IC3I.2014.7019822

Sulyman, A. I., Nassar, A. T., Samimi, M. K., MacGartney, G. R., Rappaport, T. S. \& Alsanie, A. (2014). Radio propagation path loss models for $5 \mathrm{G}$ cellular networks in the $28 \mathrm{GHz}$ and $38 \mathrm{GHz}$ millimeter-wave bands. IEEE Communications Magazine, 52(9), pp. 78-86. doi: http://dx.doi.org/10.1109/ MGOM.2014.6894456 
Swindlehurst, A. L., Ayanoglu, E., Heydari, P. \& Capolino, F. J. I. G. M. (2014). Millimeter-wave massive MIMO: The next wireless revolution? IEEE Communications Magazine, 52(9), pp. 56-62. doi: http://dx.doi.org/10.1109/ MCOM.2014.6894453

Zheng, L. \& Tse, D. N. G. (2003). Diversity and multiplexing: A fundamental tradeoff in multiple-antenna channels. IEEE Transactions on Information Theory, 49(5), pp. 1073-1096. doi: http://dx.doi.org/10.1109/TIT.2003.810646 


\section{AUTHOR}

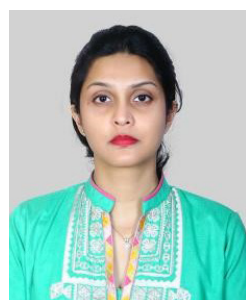

\section{Sara Bhatti}

Sara is a lecturer in Sir Syed University of Engineering and Technology. She completed her Bachelors of Engineering in Electrical and Electronic Engineering from the University of Auckland in 2002. Her areas of interest include wireless Communications, Satellite Communications and Communication Systems. 